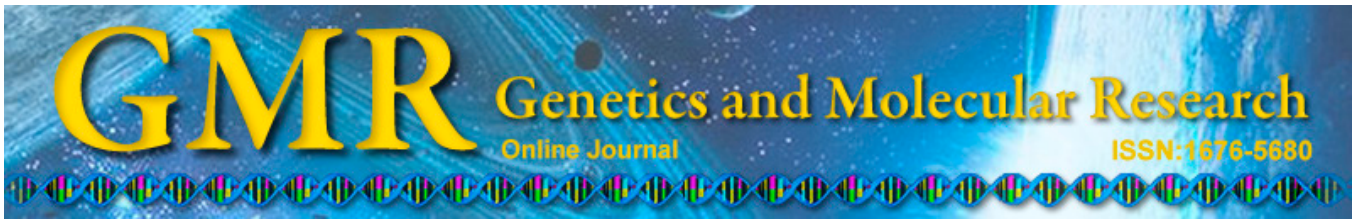

\title{
Association between macrophage migration inhibitory factor rs1007888 and GDM
}

\author{
Y. Zhan, C. Li, J. Chen, S. Yu, Q. Gao, Y.P. Wang and S.G. Liu \\ Department of Obstetrics, Affiliated Hospital of Qingdao University, \\ Qingdao, China \\ Corresponding author: Y. Zhan \\ E-mail: yingzhancn@126.com \\ Genet. Mol. Res. 14 (1): 797-804 (2015) \\ Received March 11, 2014 \\ Accepted July 3, 2014 \\ Published February 2, 2015 \\ DOI http://dx.doi.org/10.4238/2015.February.2.4
}

\begin{abstract}
We investigated the association between macrophage migration inhibitory factor $(M I F)$ rs1007888 single-nucleotide polymorphisms and the genetic susceptibility to gestational diabetes mellitus (GDM). A total of 240 GDM pregnant women (GDM group) and 330 healthy pregnant women (control group) were included in the study. Differences in the MIF rs1007888 genotype and allele frequencies and differences between fasting blood glucose, fasting insulin, homeostatic model assessment (HOMA)-insulin resistance, and HOMA- $\beta$ levels of pregnant women with different genotypes were compared. MIF genotype distributions were significantly different in the GDM group compared to the control group $(\mathrm{P}<0.05)$. No significant difference was observed in the allele distributions of MIF rs 1007888 between the GDM group and control group $(\mathrm{P}>0.05)$. GDM patients had higher fasting blood glucose, fasting insulin, and HOMA-insulin resistance levels, but lower HOMA- $\beta$ levels than normal gestational women $(\mathrm{P}<0.05)$. Fasting blood glucose, fasting insulin, and HOMA-insulin resistance in pregnant women with the GG genotype were significantly higher than those with GA and AA genotypes, while HOMA- $\beta$ in pregnant women with the GG genotype was lower (all $\mathrm{P}<0.05$ ). Our findings demonstrated the associations among MIF polymorphism rs1007888,
\end{abstract}


insulin resistance, and pancreatic $\beta$ cell functions in GDM patients. The GG genotype of MIF rs 1007888 may be a genetic susceptibility factor in the pathogenesis of GDM.

Key words: Macrophage migration inhibitory factor; Polymorphism; Gestational diabetes; rs1007888; Single-nucleotide polymorphism

\section{INTRODUCTION}

Gestational diabetes mellitus (GDM) is a common complication occurring during pregnancy (Landon and Gabbe, 2011) and refers to diabetes that develops or is discovered during gestational pregnancy. Recently, the incidence of GDM has generally increased, and elevated blood sugar (glucose) may impact maternal health and fetal development. Severe cases can be life-threatening for the mother and the child (Tam et al., 2012). Pregnancy alters a mother's internal environment, which may result in decreased insulin sensitivity; being pregnant facilitates the development of GDM (Buchanan et al., 2012). Approximately 1-14\% pregnant women suffer from GDM; additionally, the risk of postpartum type 2 diabetes (T2D) and insulin resistance after GDM is greatly increased (Ma and Chan, 2009). Currently, the pathogenesis of GDM is not clear, but GDM and T2D share several common risk factors and have a similar pathophysiology that includes glucose intolerance, insulin resistance, and impaired insulin secretion (Ryan et al., 2013). Additionally, women with a history of GDM are at an increased risk of GDM during subsequent pregnancies (Kwak et al., 2013). Several studies have shown that genetic factors play an important role in GDM occurrence (Zhang et al., 2013). Macrophage migration inhibitory factor (MIF) is a protein that can function as a cytokine, hormone, or enzyme and exhibits a variety of biological activities, playing an important role in inflammation and immunity (Grieb et al., 2010). Previous studies have shown that MIF is related to the occurrence of obesity and insulin resistance syndromes (Finucane et al., 2012), and MIF gene polymorphisms have been correlated with GDM and metabolic syndromes (Aslani et al., 2011). To further validate the genetic susceptibility to GDM based on MIF gene polymorphisms, we used polymerase chain reaction (PCR) and DNA direct sequencing technology to analyze the MIF rs 1007888 locus. We examined differences in fasting blood glucose (FBG), fasting insulin (FIN), homeostasis model assessment-insulin resistance (HOMA-IR), and homeostasis model assessment- $\beta$ cell function (HOMA- $\beta$ ) levels in pregnant women with GDM exhibiting different genotypes. The goal of this study was to determine the relationships between MIF rs1007888 single-nucleotide polymorphisms (SNPs) and GDM incidence.

\section{MATERIAL AND METHODS}

\section{Data sources}

A total of 240 GDM pregnant women, enrolled in the clinical office or who were hospitalized in the Affiliated Hospital of Qingdao University from June 2011 to July 2013, were included in the GDM group. These women were aged $27.57 \pm 6.05$ years and were at $25.17 \pm$ 4.31 gestational weeks. Gestational diabetes was diagnosed according to the American Diabetes Association guidelines: fasting, $1 \mathrm{~h}$, and $2 \mathrm{~h}$ normal glucose upper limits under fasting and administration of $75 \mathrm{~g}$ glucose were 5.1, 10.0, and $8.5 \mathrm{mM}$, respectively; women exceeding 
the upper limit were diagnosed with GDM. Additionally, 330 healthy pregnant women were included in the control group. These women were aged $27.04 \pm 5.11$ years old and were 24.21 \pm 4.34 gestational weeks. All subjects were of Han nationality, living in Northern China, and had no other complications during pregnancy. Before the pregnancy, patients in the GDM group did not have diabetes, hypertension, or other endocrine diseases. The heights and weights of all pregnant women in the prenatal group were recorded from 6-12 weeks of pregnancy, and pre-pregnancy body mass index (BMI) was calculated as weight $/$ height ${ }^{2}\left(\mathrm{~kg} / \mathrm{m}^{2}\right)$. BMI in the GDM group was $22.92 \pm 3.41$, while that in the control group was $22.78 \pm 3.21$. The 2 groups exhibited no statistically significant differences in age, gestational weeks, time of pregnancy, $\mathrm{BMI}$, or other indexes $(\mathrm{P}>0.05)$. This study was conducted in accordance with the Declaration of Helsinki. This study was conducted with approval from the Ethics Committee of Qingdao University. Written informed consent was obtained from all participants.

\section{Specimen collection and determination of related indices}

All pregnant women fasted $6 \mathrm{~h}$ before blood sampling, and $10 \mathrm{~mL}$ fasted peripheral venous blood was collected the following morning. Half of the samples were collected in $5-\mathrm{mL}$ vacuum blood collection tubes, and ethylenediaminetetraacetic acid was added to each tube as an anticoagulant and the tubes were preserved at $-80^{\circ} \mathrm{C}$ for DNA extraction. Serum was separated for the other $5 \mathrm{~mL}$ sample and stored at $-80^{\circ} \mathrm{C}$ for future experiments. FBG detection was conducted using a Hitachi 7600-2020 automatic biochemical analyzer (Hitachi, Tokyo, Japan). FIN was measured using a radioimmunoassay kit from LINCO Research, Inc. (St. Louis, MO, USA). HOMA was used to calculate the insulin resistance index (HOMA-IR) $=[\mathrm{FBG}(\mathrm{mM}) \times \mathrm{FIN}(\mathrm{mM})] / 22.5$, as well as the $\beta$-cell functional index $($ HOMA- $\beta)=20 \mathrm{x}$ FIN (mM) / [FPG (mM) - 3.5].

\section{Extraction of genomic DNA}

The phenol-chloroform method was used to extract DNA from the peripheral blood using a DNA purification kit (Tiangen Biotech Co. Ltd., Beijing, China). After determining the concentration, genomic DNA at $10-20 \mathrm{mg} / \mathrm{L}$ was stored at $-20^{\circ} \mathrm{C}$.

\section{Detection of MIF rs1007888 polymorphism}

The PCR amplification primers were designed based on relevant literature (Aslani et al., 2011) and the original DNA sequence reported in GenBank. Primer synthesis was performed by Shanghai Sunny Biotechnology Co., Ltd. (Shanghai, China). The primer sequences were: upstream primer: 5'-TTAGGGAGGGGTAAGAAC-3'; downstream primer: 5'-GAAGCCCATGTAAAAGAA-3'. The amplified fragment length was 404 base pairs. The PCR was conducted in a volume of $20 \mu \mathrm{L}$ under the following amplification conditions: predenaturation at $95^{\circ} \mathrm{C}$ for $4 \mathrm{~min}$, denaturation at $94^{\circ} \mathrm{C}$ for $30 \mathrm{~s}$, annealing at $55^{\circ} \mathrm{C}$ for $30 \mathrm{~s}$, and extension at $72^{\circ} \mathrm{C}$ for $30 \mathrm{~s}$ for 35 cycles, followed by extension at $72^{\circ} \mathrm{C}$ for $7 \mathrm{~min}$ and thermal insulation at $4{ }^{\circ} \mathrm{C}$. To detect the amplified PCR products, $2 \mu \mathrm{L}$ PCR amplified product was subjected to $1.5 \%$ agarose gel electrophoresis, with a 100-base pair ladder used to identify the PCR products. DNA direct sequencing was performed to detect the SNP in the MIF rs 1007888 locus. PCR amplification products were sequenced by Shanghai Sunsoon Biotechnology Co., 
Ltd. (Shanghai, China) by the Sanger dideoxy chain termination method. The sequencing primer sequence was 5'-TTAGGGAGGGGTAAGAAC-3', and sequencing was performed using an ANIPRISM 3730XL sequencer.

\section{Statistical analysis}

The SPSS 13.0 software was used for the statistical data processing (SPSS, Inc., Chicago, IL, USA). Measured data are reported as means \pm standard deviation, with the Student $t$-test used for the intergroup comparisons; counting data are reported as percentages, and the $\chi^{2}$ test was performed to compare differences in genotype frequencies and allele frequency distributions. The Hardy-Weinberg law of genetic equilibrium was evaluated to determine the distributions of gene frequencies in the GDM group and control group.

\section{RESULTS}

\section{Relevant clinical indicators}

FBG, FIN, and HOMA-IR levels in the GDM group were significantly higher $(\mathrm{P}<$ $0.05)$ than those in the normal group. HOMA- $\beta$ in the GDM group was significantly lower than that in the control group $(\mathrm{P}<0.05)$ (Table 1).

Table 1. Comparison of clinically relevant indicators of the 2 groups (means \pm SD).
\begin{tabular}{lccccc}
\hline Group & Cases & FBG $(\mathrm{mM})$ & FIN $(\mathrm{mM})$ & HOMA-IR & HOMA- $\beta$ \\
\hline GDM & 240 & $5.36 \pm 1.20$ & $20.45 \pm 4.84$ & $4.92 \pm 1.10$ & $193.57 \pm 78.43$ \\
Control & 330 & $4.55 \pm 0.52$ & $13.68 \pm 2.86$ & $2.61 \pm 0.62$ & $238.82 \pm 82.55$ \\
$t$ & & 8.11 & 13.69 & 7.19 & 4.70 \\
P & $<0.05$ & $<0.05$ & $<0.05$ & $<0.05$ \\
\hline
\end{tabular}

FBG = fasting blood glucose; FIN = fasting insulin; HOMA-IR = homeostasis model assessment-insulin resistance; HOMA- $\beta=$ homeostasis model assessment $\beta$ cell function.

\section{Genotype distributions and genotype frequencies}

DNA direct sequencing was used to perform the genotyping of the MIF rs 1007888 locus, and the frequencies of the GG, GA, and AA genotypes are shown in Figure 1. The results showed that the genotype distributions in the GDM group and the control group were in Hardy-Weinberg equilibrium $(\mathrm{P}>0.05)$.

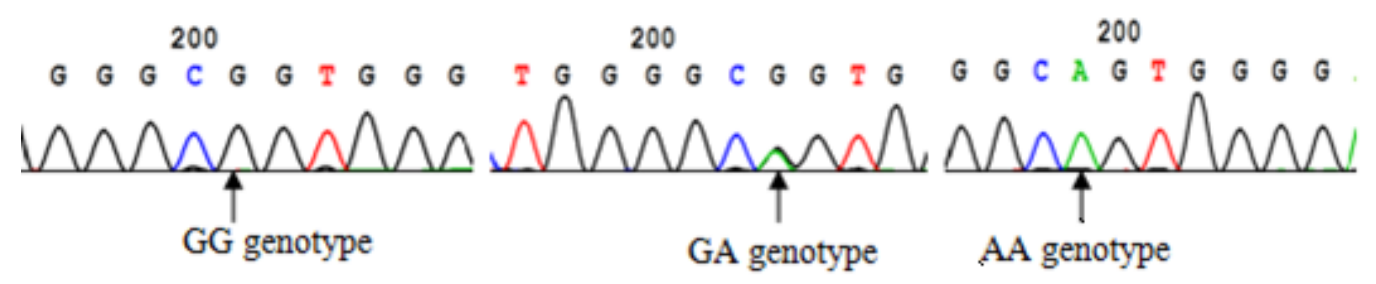

Figure 1. DNA direct sequencing results of the MIF rs1007888 SNP. 
The genotype frequencies of MIF rs1007888 GG, GA, and AA in the GDM group were $36.40,46.83$, and $16.77 \%$, respectively, while the allele frequency of $\mathrm{G}$ and A were 60.83 and $39.17 \%$, respectively. In the control group, the genotype frequencies of GG, GA, and AA were 25.04, 53.56, and $21.40 \%$, while the allele frequencies of $\mathrm{G}$ and A were 53.33 and $46.67 \%$, respectively. The difference in $M I F$ rs 1007888 genotype frequencies between the GDM and control groups was statistically significant $\left(\chi^{2}=4.26, \mathrm{P}<0.05\right)$, while the allele frequencies of $M I F$ rs 1007888 between the GDM group and control group were not statistically significant $\left(\chi^{2}=2.00, \mathrm{P}>0.05\right)$ (Table 2$)$.

\section{Table 2. Comparison of genotype distributions and genotype frequencies of the 2 groups.}

\begin{tabular}{|c|c|c|c|c|c|c|}
\hline \multirow[t]{2}{*}{ Group } & \multirow[t]{2}{*}{ Cases } & \multicolumn{3}{|c|}{ Genotype } & \multicolumn{2}{|c|}{ Allele } \\
\hline & & GG & $\mathrm{GA}+\mathrm{AA}$ & $\mathrm{AA}$ & G & A \\
\hline GDM & 240 & $87(36.40)$ & $112(46.83)$ & $41(16.77)$ & $292(60.83)$ & 188 (39.17) \\
\hline Control & 330 & $83(25.04)$ & $177(53.56)$ & $70(21.40)$ & $352(53.33)$ & 308 (46.67) \\
\hline
\end{tabular}

\section{Clinically relevant indicators among different genotypes}

Comparisons of FBG, FIN, HOMA-IR, and HOMA- $\beta$ levels between the pregnant women with GG genotypes and GA+AA genotypes revealed statistically significant differences $(\mathrm{P}<0.05)$ (Table 3$)$.

Table 3. Comparison of clinical indicators of different genotypes in the 2 groups (means \pm SD).

\begin{tabular}{lccccr}
\hline Genotype & Cases & FBG $(\mathrm{mM})$ & FIN $(\mathrm{mM})$ & HOMA-IR & HOMA- $\beta$ \\
\hline GG & 170 & $5.10 \pm 1.05$ & $18.45 \pm 8.82$ & $3.88 \pm 1.13$ & $189.63 \pm 79.45$ \\
GA+AA & 289 & $4.63 \pm 1.32$ & $13.44 \pm 4.54$ & $2.96 \pm 1.02$ & $228.73 \pm 80.62$ \\
$t$ & & 3.22 & 5.04 & 6.54 & 3.82 \\
$\mathrm{P}$ & & $<0.05$ & $<0.05$ & $<0.05$ & $<0.05$ \\
\hline
\end{tabular}

FBG = fasting blood glucose; FIN = fasting insulin; HOMA-IR = homeostasis model assessment-insulin resistance; HOMA- $\beta=$ homeostasis model assessment $\beta$ cell function.

\section{DISCUSSION}

The human MIF gene is located on chromosome $22 \mathrm{q} 11.2$, and is present as a single gene copy composed of 3 exons and 2 introns (Herder et al., 2008a; Martin et al., 2010). MIF is a 115 -amino acid protein with a relative molecular weight of $12.5 \mathrm{kDa}$. Its three-dimensional structure is composed of chains $\alpha$ and $\beta$, and shows no homology to other known proteins. Currently, the MIF molecule is not considered to be in the cytokine family, but shows multiple functions such as a cytokine, hormone, and enzymes, exhibits a variety of biological activities, and plays an important role in inflammation and immunity (Grieb et al., 2010). With the progress of the Human Genome Project, SNPs are increasingly considered to be useful for explaining individual phenotypic differences, disease susceptibility of different groups and individuals, disease tolerance towards various drugs, and response towards environmental factors. Identification of SNPs has become a primary goal of the Human Genome Project. Thus 
far, 4 SNP loci have been identified for MIF, including rs755622, rs2070766, rs2070767, and rs1007888 (Herder et al., 2008b). The rs2070766 and rs2070767 loci are present in intronic regions, where their polymorphisms do not cause mRNA shear. The MIF rs 755622 and rs1007888 loci have been thoroughly examined. The rs1007888 locus is located 3807 base pairs towards the $3^{\prime}$ end of the translation termination codon and contains the alleles $\mathrm{G}$ and A (Herder et al., 2008b). A previous study identified the GG genotype of the MIF SNP rs 1007888 was associated with myocardial infarction in Czech female patients (Tereshchenko et al., 2009). Herder et al. (2008b) also found that the G allele of the rs 1007888 locus was related to the onset of T2D in females, and was closely related to high levels of MIF in the circulation; elevated serum MIF levels were found to be related to an increase in T2D risk, suggesting that MIF has a pathogenic role in T2D.

GDM is the most common metabolic disorder during pregnancy, and shows certain similarities to the pathogenesis and clinical characteristics of T2D, such as IR (Mao et al., 2012). During pregnancy, the placenta should be secreting placental growth hormone and placental lactogen, which is antagonistic towards insulin; free fatty acids increase, and the utilization of insulin towards glucose should decrease, thereby increasing IR. This condition makes a woman more susceptible to diabetes. Recent studies have found that MIF participates in the regulation of pregnancy and the occurrence of pathological pregnancy, because MIF was expressed in the placental trophoblast cells of GDM women (Enquobahrie et al., 2009). Serum MIF in GDM patients was significantly higher than that in the normal glucose tolerance group, and was significantly and positively correlated with FBG, FIN, and HOMA-IR levels during pregnancy (Yilmaz et al., 2012).

In this study, DNA direct sequencing technology was used to analyze the genotypes and frequencies of the MIF rs1007888 locus both in a GDM group and control group, and the results showed that the genotype frequencies of GG, GA, and AA in the GDM group were $36.40,46.83$, and $16.77 \%$, respectively, while those in the control group were 25.04 , 53.56 , and $21.40 \%$. The MIF rs 1007888 genotype frequencies in the GDM group and control group exhibited statistically significant differences $(\mathrm{P}<0.05)$. The allele frequencies of MIF rs1007888 locus G and A allele frequencies in the GDM group were 60.83 and 39.17\%, while those in the control group were 53.33 and $46.67 \%$, respectively; these values were not significantly different $(\mathrm{P}>0.05)$, and the study results suggested that the GG genotype of the MIF rs 1007888 locus may be the pathogenic genotype of GDM and that the G allele may be recessive. We also found that compared with the control group, the FBG, FIN, and HOMA-IR levels in the GDM group were significantly higher, while FBG, FIN, and HOMA-IR levels for women with the MIF rs 1007888 GG genotype were significantly increased compared to those for women with the GA+AA genotype. The findings indicated that the IR degree of pregnant women carrying the GG genotype was significantly increased, which promoted the occurrence of GDM.

Studies have found that MIF has an important regulatory role in pancreatic $\beta$-cell functions (Saksida and Stosic-Grujicic, 2012). Elevated serum MIF levels were significantly negatively correlated with pancreatic $\beta$-cell functions in T2D patients through the regulation of pancreatic $\beta$-cell secretion of insulin and promotion of the uptake and utilization of glucose inside target cells to regulate the glucose metabolism. MIF may also positively regulate pancreatic $\beta$-cells to produce large amounts of MIF (Waeber et al., 1999). The regulation of MIF generation may be time- and glucose concentration-dependent (Waeber et al., 1997). GDM patients have higher levels of insulin resistance, and this insulin differentiation (Saisho 
et al., 2013) can result in pancreatic $\beta$-cell failure (Plaisance et al., 2002). The results of this study showed that compared with the control group, HOMA- $\beta$ in the GDM group was lower than that in the control group, indicating that $\beta$-cell functions in GDM patients were damaged, which is consistent with the results of other studies (Wang et al., 2013). Additionally, compared with the GA+AA genotype, HOMA- $\beta$ of the MIF-rs1007888 GG genotype was significantly reduced, indicating that the $M I F$ rs 1007888 GG genotype reduces $\beta$-cell functions. How the GG genotype promotes the occurrence and development of GDM throughout pregnancy requires further examination.

The results of this study showed that the MIF rs1007888 SNP locus may be associated with GDM incidence and that the GG genotype may increase the IR degree during pregnancy, impacting $\beta$-cell functions. This locus may be the susceptibility locus for GDM, and may be significant in the early screening and prevention of GDM.

\section{REFERENCES}

Aslani S, Hossein-nezhad A, Maghbooli Z, Mirzaei K, et al. (2011). Genetic variation in macrophage migration inhibitory factor associated with gestational diabetes mellitus and metabolic syndrome. Horm. Metab. Res. 43: 557-561.

Buchanan TA, Xiang AH and Page KA (2012). Gestational diabetes mellitus: risks and management during and after pregnancy. Nat. Rev. Endocrinol. 8: 639-649.

Enquobahrie DA, Williams MA, Qiu C, Meller M, et al. (2009). Global placental gene expression in gestational diabetes mellitus. Am. J. Obstet. Gynecol. 200: 206.e1-13.

Finucane OM, Reynolds CM, McGillicuddy FC and Roche HM (2012). Insights into the role of macrophage migration inhibitory factor in obesity and insulin resistance. Proc. Nutr. Soc. 71: 622-633.

Grieb G, Merk M, Bernhagen J and Bucala R (2010). Macrophage migration inhibitory factor (MIF): a promising biomarker. Drug News Perspect. 23: 257-264.

Herder C, Illig T, Baumert J, Müller M, et al. (2008a). Macrophage migration inhibitory factor (MIF) and risk for coronary heart disease: results from the MONICA/KORA Augsburg case-cohort study, 1984-2002. Atherosclerosis 200: 380-388.

Herder C, Klopp N, Baumert J, Müller M, et al. (2008b). Effect of macrophage migration inhibitory factor (MIF) gene variants and MIF serum concentrations on the risk of type 2 diabetes: results from the MONICA/KORA Augsburg Case-Cohort Study, 1984-2002. Diabetologia 51: 276-284.

Kwak SH, Choi SH, Jung HS, Cho YM, et al. (2013). Clinical and genetic risk factors for type 2 diabetes at early or late post partum after gestational diabetes mellitus. J. Clin. Endocrinol. Metab. 98: E744-E752.

Landon MB and Gabbe SG (2011). Gestational diabetes mellitus. Obstet. Gynecot. 118: 1379-1393.

Ma RC and Chan JC (2009). Pregnancy and diabetes scenario around the world: China. Int. J. Gynaecol. Obstet. 104 (Suppl 1): S42-S45.

Mao H, Li Q and Gao S (2012). Meta-analysis of the relationship between common Type 2 diabetes risk gene variants with gestational diabetes mellitus. PLoS One 7: e45882.

Martin RJ, Savage DA, Carson DJ, McKnight AJ, et al. (2010). Polymorphisms of the macrophage migration inhibitory factor gene in a UK population with Type 1 diabetes mellitus. Diabet. Med. 27: 143-149.

Plaisance V, Thompson N, Niederhauser G, Haefliger JA, et al. (2002). The mif gene is transcriptionally regulated by glucose in insulin-secreting cells. Biochem. Biophys. Res. Commun. 295: 174-181.

Ryan AS, McLenithan JC and Zietowski GM (2013). Accelerated metabolic susceptibility to type 2 diabetes in older women with a history of gestational diabetes. Endocr. Connect. 2: 79-86.

Saisho Y, Miyakoshi K, Ikenoue S, Kasuga Y, et al. (2013). Marked decline in beta cell function during pregnancy leads to the development of glucose intolerance in Japanese women. Endocr. J. 60: 533-539.

Saksida T and Stosic-Grujicic S (2012). Beta cell function: the role of macrophage migration inhibitory factor. Immunol. Res. 52: 81-88.

Tam WH, Ma RC, Yang X, Ko GT, et al. (2012). Cardiometabolic risk in Chinese women with prior gestational diabetes: a 15-year follow-up study. Gynecol. Obstet. Invest. 73: 168-176.

Tereshchenko I, Petrkova J, Mrazek F, Lukl J, et al. (2009). The macrophage migration inhibitory factor (MIF) gene polymorphism in Czech and Russian patients with myocardial infarction. Clin. Chim. Acta 402: 199-202. 
Waeber G, Calandra T, Roduit R, Haefliger JA, et al. (1997). Insulin secretion is regulated by the glucose-dependent production of islet beta cell macrophage migration inhibitory factor. Proc. Natl. Acad. Sci. U. S. A. 94: 4782-4787.

Waeber G, Calandra T, Bonny C and Bucala R (1999). A role for the endocrine and pro-inflammatory mediator MIF in the control of insulin secretion during stress. Diabetes Metab. Res. Rev. 15: 47-54.

Wang YH, Wu HH, Ding H, Li Y, et al. (2013). Changes of insulin resistance and $\beta$-cell function in women with gestational diabetes mellitus and normal pregnant women during mid- and late pregnant period: a case-control study. J. Obstet. Gynaecol. Res. 39: 647-652.

Yilmaz Ö, Küçük M, Kebapçilar L, Altindag T, et al. (2012). Macrophage migration-inhibitory factor is elevated in pregnant women with gestational diabetes mellitus. Gynecol. Endocrinol. 28: 76-79.

Zhang C, Bao W, Rong Y, Yang H, et al. (2013). Genetic variants and the risk of gestational diabetes mellitus: a systematic review. Hum. Reprod. Update 19: 376-390. 